\title{
Том \\ Policing orthodoxy on Wikipedia: Skeptics in action?
}

\section{Brian Martin}

Abstract

Wikipedia has been accused of being biased against challengers to scientific orthodoxy due to efforts by editors having affinities with the Skeptics movement. Examination of Wikipedia, including entries on fluoridation, the origin of AIDS and vaccination, reveals several characteristics typical of a Skeptics sensibility, including the definition of scepticism, lists of deviant ideas, derogatory labelling of heterodox viewpoints, and categories established without reference to reliable sources.

Keywords

Health communication; Representations of science and technology; Science and media

DOI

https://doi.org/10.22323/2.20020209

Submitted: 19th September 2020

Accepted: 26th January 2021

Published: 12th April 2021

We believe that organised skeptic groups are actively targeting Wikipedia articles that promote natural, non-drug therapies with which they disagree. [...] The new trick of these editors is to rewrite or entirely remove pertinent information from such articles or, worse still, delete entire articles altogether [Alliance for Natural Health, 2018].

Wikipedia currently is the area in which dogmatic skeptics are most successful and influential. [...] The situation is particularly bad in any areas to do with parapsychology, alternative and complementary medicine, and on the biography pages of scientists involved in investigating these areas [Sceptical about Skeptics, n.d.].

These are examples of claims that members of the Skeptics movement are unfairly influencing information on Wikipedia. However, those making these claims often have a commitment to the views allegedly being targeted - for example, support for natural therapies - so it might be that they are the ones who are biased. Independently substantiating their claims is difficult because Wikipedia is collectively produced and most of its editors are anonymous. This leads to the question of how the influence on Wikipedia of a particular group or movement in this case Skeptics — can be determined. 
The Skeptics movement is composed of a number of groups and networks tied together by publications - such as The Skeptic — and a common cause. Though calling themselves Skeptics (always spelled with a " $k$ " even in countries where the standard spelling is "sceptics"), their scepticism is applied asymmetrically, always against beliefs contrary to scientific and medical orthodoxy and, and their efforts are invariably against groups espousing those beliefs. The campaigning and rhetoric coming from Skeptics is often-times more vehement than anything regularly expressed by scientists in the mainstream. Most scientists get on with their work and pay little attention to views outside the scientific community. Skeptics, in contrast, have adopted the role of orthodoxy enforcers. Rather than ignoring those outside the mainstream, they seek to denigrate and discredit them.

Gieryn $[1983 ; 1995 ; 1999]$ developed the concept of "boundary work," which refers to the activities of individuals and groups to expand or maintain a boundary between science and non-science. As Gieryn [1999, p. xii] puts it, "Representations of science - where it is, and where it is not - have less to do with the cultural realities they supposedly depict, and more to do with the cultural realities they sustain." Much of this work is rhetorical: by talking about bodies of knowledge in particular ways, they are granted a specified status. In this way of thinking, what counts as science results from agreement by a sufficient number of people that it should be called science.

Most of Gieryn's case studies, for example concerning cold fusion, phrenology, composting and the science wars, involve efforts by individuals and groups inside the scientific community to police boundaries. In contrast, a considerable proportion of those involved in the Skeptics movement are not working scientists. They undertake the policing of science's boundaries and the assertion of what counts as science from a position outside the formal scientific community. Wikipedia is a domain outside of science; parts of it are about science. It is an intriguing arena for struggles over defining science carried out using rules quite different from those used within the scientific community.

This article is an attempt to make an initial and limited examination of how to determine the influence of Skeptics on Wikipedia. Some aspects of this analysis might also be applied to the study of Wikipedia bias in other areas, such as politics.

The next section provides a brief introduction to scepticism and the Skeptics movement. Following this is an introduction to Wikipedia. Then comes an analysis of several Wikipedia entries.

Scepticism and the Skeptics movement
Robert Merton, a prominent sociologist, studied the dynamics of science. In a well-known formulation [Merton, 1973], he proposed that the scientific ethos is characterised by four norms: universalism, communism (referring to common ownership), disinterestedness and organised scepticism. Whether Merton had any empirical basis for postulating these norms is debateable, but undoubtedly they have served a powerful rhetorical or ideological function. In particular, scientists have long thought of themselves as sceptics. In the rise of science in Europe, early scientists were confronted by the power of the Church, which asserted its authority over beliefs in a range of domains. Scepticism is commonly contrasted with 
dogmatism, with dogmatism linked to beliefs asserted by (non-scientific) authorities.

Later social analysts questioned whether Merton's norms actually described the practice of science [Barnes and Dolby, 1970; Mulkay, 1976]. Ian Mitroff [1974] carried out a study of moon scientists and concluded that a series of "counter-norms" were just as important as Merton's norms. The counter-norm to organised scepticism is, according to Mitroff, "organised dogmatism." This might be interpreted as suggesting that science has become a new church [Horrobin, 1969]. It is compatible with Thomas Kuhn's [Kuhn, 1963] observations about the necessary role of dogma in science.

Despite the critical analyses of Merton's norms, many scientists see themselves as sceptics, and certainly not as dogmatists. Furthermore, in much public commentary, scientists have managed to adopt the mantle of being sceptics. In some parts of the world, this remains a contrast with the dogmatism or blind faith associated with religion.

Scientists, according to Merton, are supposed to be sceptical about their own theories, or about all theories. This can be linked to the philosophy of Karl Popper [1972], who argued that science is distinguished from ideology by being able to be falsified. Scientists have taken this on board, with some of them seeing their own approach as seeking to falsify theories [Mulkay and Gilbert, 1981]. Sociologists who have studied science have argued that, in practice, scientists do not follow Popper's principle, instead retaining theories even though experimental results conflict with them.

Those who call themselves Skeptics have adopted a label associated with science but turned it in a different direction. Rather than being scientists who are sceptical of their own theories and results, or trying to falsify their own theories, Skeptics turn their attention to the ideas of others, in particular those who challenge orthodox views within science. Skeptics thus might be considered to have appropriated the rhetoric of scepticism for a crusade against heterodoxy. Rather than following some version of the scientific method, Skeptics have adopted a set of boundary-work techniques and unilaterally taken over enforcement.

Researchers in the field of science and technology studies have made in-depth analyses of the ways that scientific knowledge is created and legitimated, the discourse of scientists, and the psychology of scientists, among many other topics. A few have looked at Skeptics, for example Collins and Pinch [1982, pp. 41-46] who commented on the "scientific vigilantes" who sought to discredit research on parapsychology. Within science and technology studies, by far the most detailed analysis of the Skeptics is David Hess's book Science in the New Age, in which he examines New Agers, parapsychologists and Skeptics anthropologically, in the context of US culture. According to Hess, each of these three groups sees itself as pure and the other groups as "greedy, materialistic, egocentric, and sometimes disorderly and pathological" [Hess, 1993, p. 68]. The Skeptics see themselves as the underdogs in seeking to counter popular opinion and a mass media that panders to it for commercial reasons. Hess observed that the Skeptics used a confrontational, even aggressive approach, one with affinities to masculinity. In undertaking his analysis, Hess expanded the concept of boundary-work, seeing it as pervasive, 
used against other groups to reject their claims to knowledge and authority, and used within groups between factions with different perspectives.

Since Hess's sophisticated cultural studies analysis there has been relatively little sociological examination of the Skeptics movement. In addition, the advent of the Internet has opened new domains for struggles between Skeptics and their opponents.

Skeptics organisations in several countries are brought together by magazines, online groups (since the 1990s) and face-to-face meetings, including conferences. The most prominent is the US-based Skeptics Society, which produces a glossy magazine, The Skeptic, with regular columnists and major articles, holds an annual conference and other events. The Skeptics Society describes itself as an "educational organization that promotes science literacy and critical thinking, and investigates fringe science and paranormal claims." The topics addressed in The Skeptic give a good sense of the favourite targets for Skeptics, which include religion, intelligent design, homeopathy, therapeutic touch, post-truth and postmodernism, UFOs, flat earthers and conspiracy theories. The Skeptic is somewhat open to debate, giving space to some of the targets of its critical attention, for example those who believe in the existence of God.

Michael Shermer, the publisher and editor-in-chief of The Skeptic, is the most prominent figure in the Skeptics Society. In his 1997 book Why Do People Believe Weird Things? he tackles all sorts of "fringe" beliefs, including the paranormal, near-death experiences, alien abductions, witch crazes (including recovered memory syndrome) and Ayn Rand, with special attention to creationism and Holocaust denial.

The Skeptics movement is held together by a common set of assumptions about the world and their place in it. Skeptics assume they have access to the truth (even if it is hypothetically provisional), which is almost always based in scientific and medical orthodoxy, and that those who have different beliefs about core issues are wrong and need to be educated or countered. These assumptions come to the fore in Skeptics explanations for heterodox beliefs, which they attribute to psychological shortcomings, as in the title of Shermer's book Why Do People Believe Weird Things?

Most scientists focus on their own research projects that are built on the standard set of assumptions about knowledge and research methods. Quite a number of scientists, with appropriate credentials and publications, have challenged dominant paradigms in physics, most commonly relativity and quantum theory, but few mainstream scientists pay any attention to them or their ideas [Campanario and Martin, 2004]. A common complaint from these dissident physics researchers is that no one knowledgeable is willing to scrutinise their ideas. If mainstream scientists do not bother to examine dissident views presented by those who follow the rules of the game - writing in conventional scientific style and publishing in scientific journals - then it is not surprising that mainstream scientists have even less time for alternative views that are seen as fringe. For example, few astronomers pay any attention to astrology, even to the studies that are carried out quantitatively, much less to star-sign evaluations published in the mass media. So it might be wondered, is there any need to attack astrology, to label it pseudoscience, and expose its flaws? This role has been adopted by the Skeptics on 
their own initiative. There is no evidence that astronomers, concerned that astrologers have too much credibility, have ever called on Skeptics to mount a campaign on their behalf.

Those with Skeptics sensibilities who work in science can engage in the usual sorts of boundary work, for example seeking to prevent publication of suspect articles and deny research grants to deviant projects. However, those working in other careers cannot police science boundaries from the inside.

Furthermore, to pursue their agenda, many supporters of the Skeptics have a disadvantage: they have no special expertise for making their claims. A few are published scientists, but a science degree and even a career as a scientist do not offer special expertise for challenging the claims of parapsychologists, homeopaths or faith healers. Some members of the Skeptics may have a background in science or medicine but work in other fields. This may be the explanation for why Skeptics magazines so seldom tout their own members' domain-specific scholarly contributions. (In making these comments, I might be seen as engaging in my own boundary work in relation to non-scientist members of the Skeptics community.) There is, though, one domain where those without topic expertise can exert influence: Wikipedia.

Wikipedia is an online, open-access encyclopedia to which anyone can contribute [Jemielniak, 2014; Lih, 2009; Reagle Jr., 2010; Reagle and Koerner, 2020; Tkacz, 2015]. Traditional encyclopedias are run by editors who invite specialists to write entries in their areas of expertise; quality control is maintained by the editors and their selection of contributors. In Wikipedia, quality control is exerted by a collective process of correction and discussion. Anyone can make a change to an entry, or add a new one, and every change can be seen on the "History" tab. Usually there are several editors paying attention to changes made to any given entry, and they may revert the changes or modify them. Under the "Talk" tab, it is possible to read comments, queries and debates concerning an entry.

The brilliant conception underlying Wikipedia is that a collective process of contribution and correction, all by volunteers, can lead to high-quality information. To a considerable degree, this conception has been vindicated. Studies have shown that the accuracy of Wikipedia is not greatly different from traditional encyclopedias, and undoubtedly Wikipedia is far larger and more dynamic being revised in the light of new information, including recent events - than any other encyclopedia. It has achieved a high profile: often, when searching the web, a Wikipedia entry will appear as one of the first few items suggested.

On Wikipedia, no particular expertise is required to make a change, in other words to become an editor. Furthermore, most editors are anonymous. The history of their edits is available and some editors offer a self-description, but in many cases their real-life identities are not disclosed.

Contributions to Wikipedia are supposed to conform to a set of policies or rules, for example maintaining a neutral point of view (NPOV) and not relying on original research (NOR). With NOR, claims are supposed to be backed by credible secondary sources. For example, rather than writing that Albert Einstein made a 
pioneering contribution with his 1905 paper about special relativity, on Wikipedia it is necessary to cite a reliable source (RS) saying that Einstein's 1905 paper was pioneering. Experienced editors know how to write and maintain entries in ways that can be defended using Wikipedia rules. However, the plethora of rules creates considerable latitude for different interpretations justifying different decisions about texts.

Several features of Wikipedia make it susceptible to systematic bias. Experienced editors can be promoted to be administrators, who have greater power. Skilled editors are more familiar with the rules and can use them to counter changes they find unwelcome. When there are disagreements about entries and their content, groups with common views and whose members are skilled and persistent can get their way.

There have been a wide range of complaints about WP bias [Lovink and Tkacz, 2011; Oeberst et al., 2019; Sanger, 2020; Wikipediocracy, 2021]. Some critics have observed that most editors are male and that there is bias against women [Ford and Wajcman, 2017; Paling, 2015]; others point to racial bias. There are allegations of political bias, against the left or the right [e.g., Adler, 2020]. Of interest for the analysis here is bias against challenges to scientific orthodoxy, a charge made by a number of critics [Rotter, 2020; Sheldrake, n.d.; Ullman, 2014; Weiler, 2013].

Assessing claims about bias on Wikipedia is difficult in part because of the scale of the encyclopaedia, with millions of entries and versions in numerous languages. Assessing bias can be done on a statistical basis [Greenstein and Zhu, 2012; Greenstein and $\mathrm{Zhu}, 2018]$. Another obstacle to assessment of bias is inconsistency between different parts of Wikipedia, and yet another is the changing content.

Here, no attempt will be made to assess the scale or seriousness of any Wikipedia bias against scientific heterodoxy. The focus will be on a few topics and a few methods, in order to illustrate boundary work on Wikipedia that seems to reflect a Skeptics sensibility.

For topics, I picked three areas that I have studied in some depth: fluoridation, the origin of AIDS, and vaccination [e.g., Martin, 1991; Martin, 2010; Martin, 2018]. Having analysed the debates in these areas gives me a basis for assessing Wikipedia treatments. Wikipedia editors would probably say that, because of my studies, I am not in a position to provide a neutral assessment. That would be to apply Wikipedia rules. However, I am writing here as a social scientist, not as a Wikipedia editor, so standards and conventions common in social science apply, which means that demonstrated expertise is seen as an asset rather than a conflict of interest.

Among methods of boundary work that could be examined, I decided to look at labelling. A common method used to relegate views or fields as "not science" is to refer to them by a distinguishing label. One term often used is "pseudoscience," which refers to fields that adopt the trappings of science without the substance. Another term used similarly is "denier," which refers to someone who rejects dominant views and has no credibility for doing so, for example "climate science denier." The term "denier" draws some of its semantic potency from the term "Holocaust denier" used to refer to someone who disagrees with the consensus view that millions of Jews and others were systematically killed in Nazi Germany from 1941-1945. 
A term of special interest here is "conspiracy theory" [Dentith, 2018; Uscinski, 2018]. Defined simply, it refers to an explanation involving secret plotting, a conspiracy being a covert arrangement by two or more people to accomplish something. In this simple definition, the attacks on 11 September 2001 involved a conspiracy. The orthodox view is that 19 Muslim terrorists conspired to fly aeroplanes into major US buildings. A non-orthodox view is that members of the US government were somehow implicated in the attacks. A different definition of "conspiracy theory" specifies that the label applies only to non-orthodox views. This definition has the problem that when an explanation moves from a marginal to a dominant position, it no longer is called a conspiracy theory, even when its content is unchanged.

All three labels - pseudoscience, denier and conspiracy theory - are derogatory, at least when applied by supporters of orthodoxy [Bjerg and Presskorn-Thygesen, 2016]. Labelling using these terms is both a method of boundary work and a method of denigration. It is worth noting that it is possible to reside in the category of "not science" and to be valued; adherents to religions certainly think so. However, labels such as pseudoscience are normally intended to be stigmatising. They also serve to implicitly attribute high value to being scientific.

In the next section, I examine some features of Wikipedia's treatment of challengers to scientific orthodoxy, including the use of stigmatising labels in the English-language Wikipedia entries for fluoridation, the origin of AIDS, and vaccination.

\section{Wikipedia treatment of scientific and medical heterodoxy}

Wikipedia hosts a "project" titled "Wikiproject Skepticism" (https://en.wikipedia.org/wiki/Wikipedia:WikiProject_Skepticism) that encapsulates the sort of boundary work undertaken by members of the Skeptics movement. ${ }^{1}$ On the project page there is a link to a list of topics (https://en.wikipedia.org/wiki/Wikipedia:WikiProject_Skepticism/List). Its first two sections, the main ones, are "valid science" and "pseudoscience." The implication is that Wikipedia, as a collectively produced representation of knowledge, has the authority and the ability to distinguish "valid science" from what doesn't constitute "valid science," labelled here as pseudoscience.
It is also revealing to look at Wikipedia's description of "scientific skepticism":

Scientific skepticism or rational skepticism (also spelled scepticism), sometimes referred to as skeptical inquiry, is an epistemological position in which one questions the veracity of claims lacking empirical evidence. In practice, the term most commonly references the examination of claims and theories that appear to be beyond mainstream science, rather than the routine discussions and challenges among scientists.

What this description lacks is evidence that philosophers, sociologists and others who have written extensively about scepticism in science direct their attention to claims outside of mainstream science. As noted earlier, Merton [1973] posited

\footnotetext{
${ }^{1}$ All statements about Wikipedia categories and quotes from Wikipedia refer to the January 2021 English-language Wikipedia.
} 
"organised scepticism" as one of the four norms of science that apply within the mainstream scientific community. The Wikipedia sentence quoted above about how "scientific skepticism" is applied "in practice" best applies to the Skeptics movement.

Wikipedia's compendium of alternative medicine includes a huge number of modalities and treatments, from acupuncture to yoga as therapy. Wikipedia includes a "sidebar" on alternative medicine. When a topic is included in the sidebar - which is edited and discussed on a separate page - then the sidebar appears on the topic page.

Among the categories included in the sidebar on "Alternative medicine" is "conspiracy theories." It so happens that three of the topics contained in the sidebar on conspiracy theories are ones about which I have written scholarly analyses. Accordingly, I look at these three topics, searching especially for the rationale for labelling them conspiracy theories, and compare Wikipedia's approach to that of the Encyclopædia Britannica.

\section{Water fluoridation controversy}

Fluoridation refers to adding compounds containing the element fluorine to public water supplies. In solution, fluorine becomes its ionised form fluoride. The aim of fluoridation is to reduce the incidence of tooth decay in people, especially children, who drink the water. Fluoridation was first widely introduced in the United States in the 1950s and then was taken up by governments in a number of other countries.

From its earliest days, fluoridation was controversial. Proponents have argued that it greatly reduces tooth decay and has few if any adverse effects. Critics have claimed that fluoridation can lead to skeletal fluorosis, reductions in IQ and a number of other adverse effects. Critics have also questioned the scale of the benefits. The controversy also has ethical and political dimensions. Proponents say fluoridation is especially important for people unable to afford dental care, whereas critics say fluoridation is compulsory medical treatment with an uncontrolled dose. Proponents usually say decisions should be made by governments on advice from health experts whereas critics often support citizen participation, for example via referenda [Freeze and Lehr, 2009; Martin, 1991].

Wikipedia has an entry on water fluoridation, which addresses many matters such as evidence, mechanism, alternatives, history and economics. There is a separate entry titled "Water fluoridation controversy," which discusses the antifluoridation movement. On 6-7 November 2016, this latter entry was added to the sidebar about "Alternative medicine" in its subcategory of "Conspiracy theories," implying that antifluoridationism is a conspiracy theory.

In the history of the fluoridation controversy, there have been a few opponents who have alleged that certain groups have promoted fluoridation to serve their vested interests. However, based on my studies of the controversy [Martin, 1991], these views have always been marginal. Most antifluoridation campaigners are driven by concerns about adverse health effects and the imposition of a semi-compulsory treatment at an uncontrolled dose. 
Wikipedia's entry on the water fluoridation controversy contains some discussion of conspiracy theories, but no comment about whether these views are prevalent or important among antifluoridation campaigners and supporters. Furthermore, there is no attempt to say that antifluoridationism is itself a conspiracy theory. The point here is that the conspiracy-theory tag has been applied by some editors of the Alternative Medicine sidebar without sufficient justification in the relevant entry.

Interestingly, the mainstream history of promotion of fluoridation can be read as a conspiracy by a number of Wisconsin dentists who campaigned in the face of official resistance to adding fluoride to public water supplies [McNeil, 1957]. In the early years, support for fluoridation challenged mainstream views, but because the conspiracy-theory label is only applied to current heterodoxy, profluoridationism has never been described as a conspiracy theory — at least not in Wikipedia.

The Encyclopædia Britannica [2021] has some information about fluoridation. It is one-sided, entirely supportive of fluoridation, but has no mention of conspiracy theories.

\section{OPV-AIDS hypothesis}

The standard view about the origin of AIDS is that simian immunodeficiency viruses (SIVs) from monkeys or chimpanzees entered humans, becoming HIVs, and became transmissible. The question is how this happened. According to the cut-hunter theory, when a hunter in Africa was butchering a chimp, chimp blood accidentally got into a wound, allowing the chimp SIV to infect the hunter [de Sousa et al., 2010].

A different view is that some polio vaccines - which for decades were grown on monkey kidneys - were prepared on chimp kidneys. These vaccines, given to nearly a million people in central Africa in the late 1950s, enabled SIVs to enter humans. This is called the OPV theory, referring to oral polio vaccines: the vaccines were given orally [Hooper, 2000].

In January 2020, the Wikipedia entry named "OPV-AIDS hypothesis" was renamed "OPV-AIDS conspiracy theory" and the alternative medicine sidebar was added, with OPV-AIDS listed in its conspiracy-theory category. On the talk page, an editor gave the reason: "The concept of intentional creation of HIV is fringe." This was based on a misunderstanding of the OPV theory, which is quite different from the view that HIV was created in a biological warfare lab. In this instance, a theory was incorrectly classified - and stigmatised — due to ignorance by Wikipedia editors. After some months, the name of the entry was changed to "Oral polio vaccine AIDS hypothesis," thereby rectifying the misleading label.

The Encyclopædia Britannica has considerable information about AIDS, but just two paragraphs about the origin of AIDS, presenting the orthodox cut-hunter view. It does not mention the OPV theory. 
Vaccination, as a means to prevent and control infectious diseases, is universally endorsed by medical authorities. Nevertheless, there are some critics of vaccination or of particular vaccines, including some scientists and doctors [e.g. Gøtzsche, 2020]. Some parents have reservations about the recommended vaccination schedule, and prefer their children to have just some of the recommended vaccines, or to have them spaced out [Reich, 2016]. In much writing about vaccination in the mass and social media, the diversity of people's concerns is ignored and anyone who questions government recommendations is labelled "anti-vaccination" or "anti-vax." On the other hand, some social scientists and medical scientists are sensitive to the complexity of the issue and avoid the term "anti-vax," at least in publications.

On Wikipedia, the "anti-vaccination" label is applied. The "anti-vaccination movement" was added to the sidebar on alternative medicine in November 2016, and more recently appears on the entry for "Vaccine hesitancy." According to the entry, vaccine hesitancy is "also known as 'anti-vaccination' or 'anti-vax'." In this entry, there are a few mentions of conspiracy theories, though put in context with the statement that "While some anti-vaccinationists openly deny the improvements vaccination has made to public health, or succumb to conspiracy theories, it is much more common to cite concerns about safety." [endnotes omitted]. The mentions of conspiracy theories in the entry do not constitute a case for what the sidebar implies, namely that anti-vaccination is itself a conspiracy theory. The conspiracy-theory label thus might be interpreted primarily as a discrediting device.

The Encyclopædia Britannica has many entries concerning vaccination. None of them mentions conspiracy theories.

\section{Assessment}

In summary, on three topics covered by Wikipedia - fluoridation, the origin of AIDS and vaccination - critics of scientific and medical orthodoxy have been labelled conspiracy theorists. In each case, this label seems more stigmatising than descriptive, given the lack of justification for the label in the relevant entries. The Encyclopædia Britannica does not associate any of these topics with conspiracy theories. Compared with Britannica, Wikipedia editors seem to have gone to a lot of trouble to identify and discredit so-called fringe beliefs in science and medicine. In this treatment, the outcome of Wikipedia editing seems to be aligned with the agenda of the Skeptics movement.

The boundary work involved on these topics is quite specific. In the usual varieties of boundary work, entire fields, such as parapsychology and astrology, are categorised as non-science. For the Skeptics movement, and at least some Wikipedia entries, this boundary work extends to any viewpoint that clashes with current scientific and medical orthodoxy.

This examination reveals a feature of Wikipedia that seldom receives attention. Contributions to Wikipedia are supposed to be based on reliable sources (RS), 
namely credible or authoritative sources of information outside Wikipedia itself. Yet it seems that in setting up categories - for example "alternative medicine" or "pseudoscience" - on Wikipedia, there is no requirement or expectation that these be based on RS. This means that editors can assert their own views about classification systems quite independently of whether the same classifications are used, much less justified, outside Wikipedia. For example, on 30 July 2019, administrator Guy set up a category, "Vaccine hesitancy," without any reference to the way vaccine hesitancy is defined or described by the World Health Organization or by scholars in the vaccine field. The category lists four sub-categories: anti-vaccination activists, anti-vaccination media, anti-vaccination organizations and orthopathy, plus 18 Wikipedia pages, including topics and individuals. Neither the talk nor the history tabs reveal any justification for this categorisation, much less any RS. The alternative medicine sidebar is another example of categorisation not backed by RS.

Establishing categories is a powerful way of shaping perceptions and constraining contributions. Wikipedia's lack of an expectation for having reliable sources for its system of categories is a gap in its architecture and rules that seems to have been filled by editors with Skeptic sensibilities, and no doubt by others in different domains.

\section{Conclusion}

Boundary work refers to efforts to demarcate one area from another. In science, boundary work is most commonly carried out by scientists to distinguish science from non-science, in a situation in which science is seen as intrinsically more valuable. The examination here is of scientific boundary work carried out by groups operating outside the scientific domain. Specifically, the Skeptics movement operates almost like a vigilante group, attacking groups deemed to be non-scientific far more than do most scientists.

To look more closely at the influence of the Skeptics movement, the domain of Wikipedia was examined. Unlike traditional encyclopedias, in which different entries are written by topic specialists, Wikipedia offers the possibility for individuals without topic expertise to have an impact on a range of topics. Wikipedia thus is a fertile field for those with a Skeptics sensibility, whether or not they coordinate their actions, to influence entries on a range of topics in the same general direction.

One of the methods used on Wikipedia is to apply labels that, in the guise of being descriptions, are stigmatising. Skeptics regularly use the term "pseudoscience" and "conspiracy theory" in a derogatory fashion. When these terms are used systematically on Wikipedia, this is compatible with there being an influence from a Skeptics perspective.

The dynamics of Wikipedia are quite different from those affecting social media. On social media, it is easy to set up blogs, Facebook pages and the like. Skeptics might want to censor the online presence of those they oppose, but have no reliable way to do so. Critics of fluoridation and vaccination have well established portals on the World Wide Web [e.g. Helmi, Spinella and Seymour, 2018]. The key here is that social media facilitate the expression of many voices. In contrast, Wikipedia is built around presenting a single voice, seen by readers as the voice of Wikipedia 
itself. The structure of Wikipedia, like that of traditional encyclopaedias, does not allow rival entries. It is in this context that Wikipedia editors sharing a similar orientation can have a great influence on the choice, content and expressive characteristics of Wikipedia entries, something not possible in social media domains.

This is a preliminary study, necessarily so because of the lack of recent studies of the Skeptics movement by social scientists, the vast scale of Wikipedia, the anonymity of many of its editors and the contested and ever-changing content of Wikipedia entries. Nevertheless, this investigation shows the value of looking at the role of groups outside science in undertaking boundary work. It also shows the potential of examining Wikipedia for evidence of such boundary work. Despite the challenges posed by editor anonymity and content variability, Wikipedia provides some compensating advantages for social analysts: edit histories and talk pages offer information about the transformations of particular entries that is usually impossible to access in traditional encyclopedias.

The approach used here involves looking for parallels - or homologies or congruence - between Wikipedia entries and characteristic techniques used by Skeptics. Specifically, (1) Wikipedia's description of scientific scepticism is more typical of the view of Skeptics than usage within the scientific community or the social studies of science; (2) Wikipedia's inclusion of lists of deviant ideas and practices is closer to the methods of Skeptics organisations than to the practice in traditional encyclopedias; (3) Wikipedia's labelling of certain views as pseudoscience and conspiracy theories is characteristic of approaches used by Skeptics; and (4) Wikipedia's categories, established without reliance on reliable sources, are congruent with Skeptic orientations. This does not prove that Skeptics are shaping Wikipedia but is compatible with that possibility.

The same sort of approach might be used to study other sources of bias on Wikipedia. For example, it would be revealing to study the use of derogatory language in case studies from a variety of areas, for example history, medicine, environment, politics and religion. As well, other indicators, in addition to language, could be used, for example choices of sources cited and addition or deletion of text.

Social science investigators might consider the possibility of learning about Wikipedia by becoming an editor. In the tradition of action research, engagement in the domain being studied can provide insights not available to a non-participating researcher. Whether or not a researcher could make any lasting change to Wikipedia entries, there are plenty of outlets for publication outside the Wikipedia domain.

Acknowledgments Thanks to Lee Basham, Daniel Beckman, Kurtis Hagen, David Hess, Jim Page and an anonymous reviewer for valuable comments. 
Adler, T. D. (13th July 2020). ‘Wikipedia editors smeared Mark Levin in multiyear campaign'. Breibart.com. URL: https://www . breitbart.com/tech/2020/07/13/w ikipedia-editors-smeared-mark-levin-in-multiyear-campaign/.

Alliance for Natural Health (12th December 2018). Wikipedia censorship of natural, non-drug therapies. URL: https://www . anhinternational .org/2018/12/12/wiki pedia-censorship-of-natural-non-drug-therapies/.

Barnes, S. B. and Dolby, R. G. A. (1970). 'The Scientific Ethos: A deviant viewpoint'. European Journal of Sociology 11 (1), pp. 3-25. https://doi.org/10.1017/s0003975600001934.

Bjerg, O. and Presskorn-Thygesen, T. (2016). 'Conspiracy Theory: Truth Claim or Language Game?' Theory, Culture E Society 34 (1), pp. 137-159. https://doi.org/10.1177/0263276416657880.

Campanario, J. M. and Martin, B. (2004). 'Challenging dominant physics paradigms'. Journal of Scientific Exploration 18 (3), pp. 421-438.

Collins, H. M. and Pinch, T. (1982). Frames of Meaning: The Social Construction of Extraordinary Science. Reprinted in 2009. London, U.K.: Routledge.

de Sousa, J. D., Müller, V., Lemey, P. and Vandamme, A.-M. (2010). 'High GUD incidence in the early 20th Century created a particularly permissive time window for the origin and initial spread of epidemic HIV strains'. PLoS ONE 5 (4), e9936.

Dentith, M. R. X., ed. (2018). Taking conspiracy theories seriously. Lanham, MD, U.S.A.: Rowman \& Littlefield.

Encyclopædia Britannica (2021). Chicago, IL, U.S.A.: Britannica Group.

Ford, H. and Wajcman, J. (2017). "“Anyone can edit", not everyone does: Wikipedia's infrastructure and the gender gap'. Social Studies of Science 47 (4), pp. 511-527. https://doi.org/10.1177/0306312717692172.

Freeze, R. A. and Lehr, J. H. (2009). The Fluoride Wars: How a modest public health measure became America's longest-running political melodrama. Hoboken, NJ, U.S.A.: John Wiley \& Sons, Inc. https : //doi .org/10.1002/9780470463710.

Gieryn, T. F. (1983). 'Boundary-Work and the Demarcation of Science from Non-Science: Strains and Interests in Professional Ideologies of Scientists'. American Sociological Review 48 (6), pp. 781-795. https://doi.org/10.2307/2095325.

- (1995). 'Boundaries of science'. In: Handbook of science and technology studies. Ed. by S. Jasanoff, G. E. Markle, J. C. Peterson and T. Pinch. Thousand Oaks, CA, U.S.A.: Sage, pp. 393-443. https: //doi .org/10.4135/9781412990127.n18.

- (1999). Cultural Boundaries of Science: Credibility on the Line. Chicago, U.S.A.: University of Chicago Press. URL: https://www . press . uchicago . edu/ucp/book s/book/chicago/C/bo3642202.html.

Gøtzsche, P. C. (2020). Vaccines: Truth, lies and controversies. Copenhagen, Denmark: People's Press.

Greenstein, S. and Zhu, F. (2012). 'Is Wikipedia biased?' American Economic Review 102 (3), pp. 343-348. URL: https: //www. jstor.org/stable/23245554.

Greenstein, S. and Zhu, F. (2018). 'Do Experts or Crowd-Based Models Produce More Bias? Evidence from Encyclopedia Britannica and Wikipedia'. MIS Quarterly 42 (3), pp. 945-959. https : //doi.org/10.25300/misq/2018/14084.

Helmi, M., Spinella, M. K. and Seymour, B. (2018). 'Community water fluoridation online: an analysis of the digital media ecosystem'. Journal of Public Health Dentistry 78 (4), pp. 296-305. https://doi.org/10.1111/jphd.12268. 
Hess, D. J. (1993). Science in the New Age: The paranormal, its defenders and debunkers, and American culture. Madison, U.S.A.: University of Wisconsin Press.

Hooper, E. (2000). The river: A journey to the source of HIV and AIDS. Boston, U.S.A.: Little, Brown.

Horrobin, D. F. (1969). Science is God. Aylesbury, U.K.: Medical and Technical Publishing Company.

Jemielniak, D. (2014). Common Knowledge? An ethnography of Wikipedia. Stanford, CA, U.S.A.: Stanford University Press. https://doi.org/10.11126/stanford/9780804789448.001.0001.

Kuhn, T. S. (1963). 'The function of dogma in scientific research'. In: Scientific change: Historical studies in the intellectual, social and technical conditions for scientific discovery and technical invention, from antiquity to the present. Ed. by A. C. Crombie. London, U.K.: Heinemann, pp. 347-369.

Lih, A. (2009). The Wikipedia revolution: How a bunch of nobodies created the world's greatest encyclopedia. London, U.K.: Aurum.

Lovink, G. and Tkacz, N., eds. (2011). Critical point of view: A Wikipedia reader. Amsterdam, Netherlands: Institute of Network Cultures.

Martin, B. (1991). Scientific knowledge in controversy: The social dynamics of the fluoridation debate. Albany, U.S.A.: State University of New York Press.

- (2010). 'How to Attack a Scientific Theory and Get Away with It (Usually): The Attempt to Destroy an Origin-of-AIDS Hypothesis'. Science as Culture 19 (2), pp. 215-239. https://doi.org/10.1080/09505430903186088.

- (2018). Vaccination panic in Australia. Sparsnäs, Sweden: Irene Publishing.

McNeil, D. R. (1957). The fight for fluoridation. New York, U.S.A.: Oxford University Press.

Merton, R. K. (1973). 'The normative structure of science'. In: The Sociology of Science: Theoretical and Empirical Investigations. Chicago, IL, U.S.A.: University of Chicago Press, pp. 267-278.

Mitroff, I. I. (1974). The subjective side of science. Amsterdam, Netherlands: Elsevier.

Mulkay, M. J. (1976). 'Norms and ideology in science'. Social Science Information 15 (4/5), pp. 637-656.

Mulkay, M. and Gilbert, G. N. (1981). 'Putting Philosophy to Work: Karl Popper's Influence on Scientific Practice'. Philosophy of the Social Sciences 11 (3), pp. 389-407. https://doi.org/10.1177/004839318101100306.

Oeberst, A., Beck, I., Matschke, C., Ihme, T. A. and Cress, U. (2019). ‘Collectively biased representations of the past: Ingroup Bias in Wikipedia articles about intergroup conflicts'. British Journal of Social Psychology 59 (4), pp. 791-818. https://doi.org/10.1111/bjso.12356.

Paling, E. (21st October 2015). 'Wikipedia's hostility to women'. The Atlantic. URL: https://www. theatlantic .com/technology/archive/2015/10/how-wiki pedia-is-hostile-to-women/411619/.

Popper, K. R. (1972). Objective knowledge: An evolutionary approach. Oxford, U.K.: Clarendon Press.

Reagle Jr., J. M. (2010). Good faith collaboration: The culture of Wikipedia. Cambridge, MA, U.S.A.: MIT Press.

Reagle, J. and Koerner, J., eds. (2020). Wikipedia @ 20: Stories of an Incomplete Revolution. Cambridge, MA, U.S.A.: MIT Press. 
Reich, J. A. (2016). Calling the shots: Why parents reject vaccines. New York, U.S.A.: New York University Press.

URL: https://www . jstor.org/stable/j.ctt1803zjf.

Rotter, C. (9th March 2020). ‘Delingpole: Wikipedia airbrushes list of climate sceptic scientists out of history'. Watts Up With That?

URL: https://wattsupwiththat.com/2020/03/09/delingpole-wikipedia-air brushes-list-of-climate-sceptic-scientists-out-of-history/.

Sanger, L. (14th May 2020). 'Wikipedia is badly biased'. Larrysanger.org. URL: https://larrysanger.org/2020/05/wikipedia-is-badly-biased/.

Sceptical about Skeptics (n.d.). Wikipedia. URL: https://www . skepticalaboutskept ics.org/wikipedia-captured-by-skeptics/.

Sheldrake, R. (n.d.). Wikipedia under threat. URL: https://www. sheldrake.org/essays/wikipedia-under-threat.

Shermer, M. (1997). Why people believe weird things? Pseudoscience, superstition, and other confusions of our time. New York, NY, U.S.A.: W. H. Freeman/ Times Books / Henry Holt \& Co.

Tkacz, N. (2015). Wikipedia and the politics of openness. Chicago, U.S.A.: University of Chicago Press.

Ullman, D. (10th October 2014). ‘Dysfunction at Wikipedia on homeopathic medicine'. HuffPost.

URL: http://huffpost.com/entry/dysfunction-at-wikipedia-_b_5924226.

Uscinski, J. E., ed. (2018). Conspiracy Theories and the People Who Believe Them. New York, U.S.A.: Oxford University Press. https://doi.org/10.1093/oso/9780190844073.001.0001.

Weiler, C. (2013). Psi wars: TED, Wikipedia and the battle for the Internet. U.S.A.: White Crow Books.

Wikipediocracy (2021). Wikipediocracy. URL: http://wikipediocracy.com.

Author

\section{How to cite}

(C) The Author(s). This article is licensed under the terms of the Creative Commons Attribution - NonCommercial - NoDerivativeWorks 4.0 License.

ISSN 1824-2049. Published by SISSA Medialab. jcom.sissa.it 